\title{
Five-wavelength optical-resolution photoacoustic microscopy of blood and lymphatic vessels
}

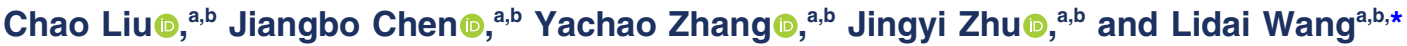 \\ ${ }^{a}$ City University of Hong Kong, Department of Biomedical Engineering, Kowloon, Hong Kong SAR, China \\ ${ }^{\mathrm{b}}$ City University of Hong Kong, Shenzhen Research Institute, Shenzhen, China
}

\begin{abstract}
Optical-resolution photoacoustic microscopy (OR-PAM) has been developed for anatomical, functional, and molecular imaging but usually requires multiple scanning for different contrasts. We present five-wavelength OR-PAM for simultaneous imaging of hemoglobin concentration, oxygen saturation, blood flow speed, and lymphatic vessels in single raster scanning. We develop a five-wavelength pulsed laser via stimulated Raman scattering. The five pulsed wavelengths, i.e., 532, 545, 558, 570, and 620/640 nm, are temporally separated by several hundreds of nanoseconds via different optical delays in fiber. Five photoacoustic images at these wavelengths are simultaneously acquired in a single scanning. The 532- and $620 / 640-\mathrm{nm}$ wavelengths are used to image the blood vessels and dye-labeled lymphatic vessels. The blood flow speed is measured by a dual-pulse method. The oxygen saturation is calculated and compensated for by the Grüneisen-relaxation effect. In vivo imaging of hemoglobin concentration, oxygen saturation, blood flow speed, and lymphatic vessels is demonstrated in preclinical applications of cancer detection, lymphatic clearance monitoring, and functional brain imaging.
\end{abstract}

Keywords: optical-resolution photoacoustic microscopy; stimulated Raman scattering; single-shot; multi-contrast microscopy; early cancer detection.

Received Oct. 12, 2020; revised manuscript received Nov. 26, 2020; accepted for publication Dec. 11, 2020; published online Jan. 4, 2021.

(C) The Authors. Published by SPIE and CLP under a Creative Commons Attribution 4.0 Unported License. Distribution or reproduction of this work in whole or in part requires full attribution of the original publication, including its DOI.

[DOI: 10.1117/1.AP.3.1.016002]

\section{Introduction}

In vivo imaging of blood vessels and lymphatic vessels is important for monitoring physiological parameters and early diagnosing of fatal diseases. ${ }^{1-3}$ The blood and lymphatic vessels are two sets of circulatory networks that have distinct but interdependent functions. Lymphatic vessels are found in most tissues and play an important role in many physiological processes. ${ }^{4}$ The blood circulation supplies oxygen and nutrients, removes waste, and is highly associated with the lymphatic circulation. Recent research has shown that blood flow, local oxygen content, and lymphatic vessels are important factors in tumor growth and metastasis. ${ }^{5-14}$ Simultaneous imaging of blood and lymphatic vessels is of great value in disease diagnosis and treatment. ${ }^{3}$ Most existing bioimaging techniques are limited to either low spatial/temporal resolution or unable to simultaneously image multi-contrasts in the blood and lymphatic

*Address all correspondence to Lidai Wang, lidawang@ cityu.edu.hk vessels. A high-resolution multi-contrast imaging technique that can image both the blood and the lymphatic vessels is highly needed.

Recently, optical-resolution photoacoustic microscopy (ORPAM) has been developed for in vivo imaging of hemoglobin concentration $\left(C_{\mathrm{Hb}}\right)$, oxygen saturation $\left(\mathrm{sO}_{2}\right)$, blood flow speed $(v)$, metabolic rate of oxygen, and lymphatic vessels. ${ }^{15-43}$ Although promising, limited by the laser source, simultaneous imaging of blood and lymphatic vessels has not been implemented in OR-PAM. In a single scanning, OR-PAM can only quantify one or two contrasts. Repetitive scanning must be done to map multiple functional or molecular contrasts, which lowers the throughput and temporal correlation among different quantities.

Here, we present five-wavelength OR-PAM that can image the blood and lymphatic vessels in a single scanning. Multiple contrasts, including hemoglobin concentration, blood flow, oxygen saturation, and exogenous dyes, can be quantified. Based on the stimulated Raman scattering (SRS) effect, ${ }^{44}$ we develop 
a five-wavelength $(532,545,558,570$, and $620 / 640 \mathrm{~nm})$ nanosecond pulsed laser. At each scanning spot, five A-lines at these wavelengths are sequentially acquired. The 532-nm wavelength is used to image hemoglobin concentration; the 532- and $545-\mathrm{nm}$ wavelengths are used to measure the blood flow speed using a dual-pulse method; the 532-, 545-, 558-, and 570-nm wavelengths are used to determine the oxygen saturation $\left(\mathrm{sO}_{2}\right)$; and the $620 / 640-\mathrm{nm}$ wavelength is used to image the dyelabeled lymphatic vessels. Using the five-wavelength OR-PAM system, we demonstrate high-resolution multi-contrast in vivo imaging of blood and lymphatic vessels in tumor growth, lymphatic dye clearance, and brain function monitoring.

\section{Methods}

\subsection{Five-Wavelength Pulsed Laser}

The five-wavelength pulsed laser needs to have sufficient pulse energy for each wavelength and nanosecond wavelength switching time. Figure 1 shows a schematic of the laser and the imaging probe. We use a pulsed laser (532-nm wavelength, VPFL-G-20, Spectra-Physics) to pump the other wavelengths via the SRS effect in optical fibers. The Raman frequency shift of the silica fiber is $\sim 13.2 \mathrm{THz}$, corresponding to a $13-\mathrm{nm}$ wavelength shift near $532 \mathrm{~nm}$. The five wavelengths are 532, $545,558,570$, and $620 / 640 \mathrm{~nm}$. The five pulses are temporally separated with different optical delays in the fiber. The pulse energy on the sample surface for each wavelength is above $90 \mathrm{~nJ}$.

In the SRS shifter, two polarizing beam splitters $\left(\mathrm{PBS}_{1}\right.$ and $\mathrm{PBS}_{2}$, PBS251, Thorlabs Inc.) and one $10 / 90$ beam splitter (BS, BSN10, Thorlabs Inc.) split the pump laser beam into four paths, i.e., a 532-nm direct path, a 545-nm Raman path, a 620/640-nm Raman path, and a 558/570-nm Raman path. The pump pulse width is $7 \mathrm{~ns}$. The total pump energy is $23.5 \mu \mathrm{J}$ per pulse. The incident pulse energies for the split paths are $0.25 \mu \mathrm{J}$ for the $532-\mathrm{nm}$ path, $2.25 \mu \mathrm{J}$ for the $545-\mathrm{nm}$ path, $8 \mu \mathrm{J}$ for the $620 / 640-\mathrm{nm}$ path, and $13 \mu \mathrm{J}$ for the $558 / 570-\mathrm{nm}$ path. Half-wave plates ( $\mathrm{HWP}_{1-6}$, WPH10E-532, Thorlabs Inc.) are used to control the polarization so that the SRS efficiency can be adjusted. Neutral density filters $\left(\mathrm{NDF}_{1-5}\right.$, NDC-50C-2, Thorlabs Inc.) are used to adjust the laser pulse energies.

The 532-nm path is coupled into the OR-PAM probe with minimal delay. The $545-\mathrm{nm}$ path uses a $25-\mathrm{m}$ polarizationmaintaining single-mode fiber (PM-S405-XP, Nufern) to shift the wavelength and delay the pulse by $121 \mathrm{~ns}$. A long-pass filter (LPF, RET537lp, Chroma) is used to select the 545-nm wavelength. In the 620/640-nm Raman path, a 50-m fiber (PM-S405-XP, Nufern) is used to generate the 620/640-nm wavelength and delay it by $243 \mathrm{~ns}$. In the 558/570-nm Raman path, the pump beam is coupled into a $100-\mathrm{m}$ graded-index multimode fiber (MMF) [GIMMSC (50/125)HT, Fibercore] to generate the 558- and 570-nm wavelengths and delay them by

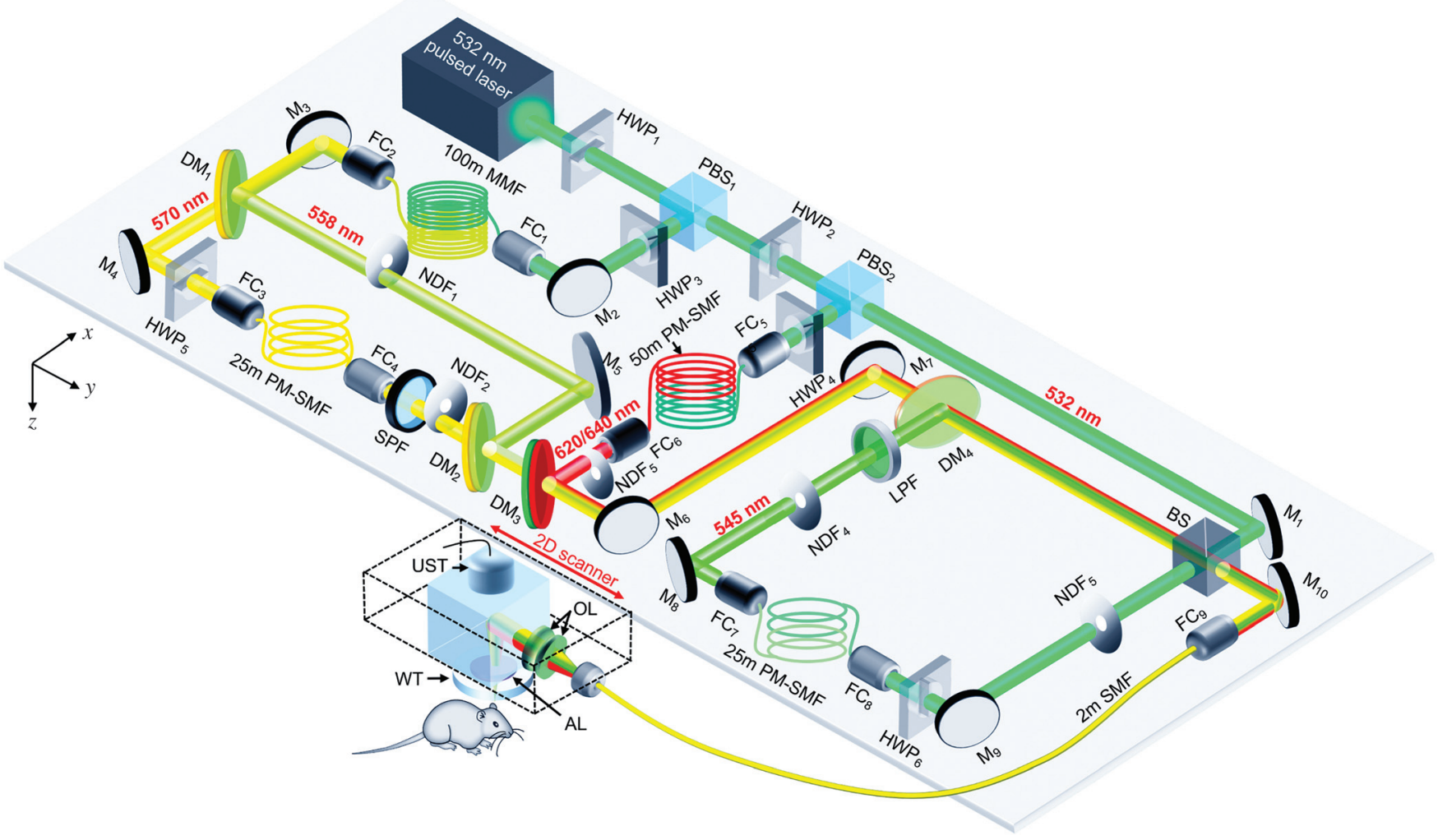

Fig. 1 Schematic of five-wavelength OR-PAM. AL, acoustic lens; $B S$, beam splitter; R/T, 10/90; $\mathrm{DM}_{1}, \mathrm{DM}_{2}, 565-\mathrm{nm}$ long-pass dichroic mirror; $\mathrm{DM}_{3}, 600$-nm short-pass dichroic mirror; $\mathrm{DM}_{4}, 550-\mathrm{nm}$ long-pass dichroic mirror; FC, fiber coupler; $\mathrm{HWP}_{1-6}$, half-wave plate; LPF, 537-nm long-pass filter; $\mathrm{M}_{1-10}$, mirror; MMF, graded-index multimode fiber; $\mathrm{NDF}_{1-5}$, neutral density filter; OL, optical lens; PBS $_{1-2}$, polarizing beam splitter; PM-SMF, polarization-maintaining single-mode fiber; SPF, 580-nm short-pass filter; UST, ultrasonic transducer; WT, water tank. 
$486 \mathrm{~ns}$. Because the multimode fiber has a larger core diameter $(50 \mu \mathrm{m})$, it has a higher SRS threshold and thus can output higher pulse energy than a single-mode fiber at the same length. ${ }^{45}$ Furthermore, the MMF can generate the SRS wavelengths with at least $75 \%$ output energy in the fundamental mode. ${ }^{46} \mathrm{~A}$ longpass dichroic mirror $\left(\mathrm{DM}_{1}, \mathrm{~T} 565 \mathrm{lpxr}-\mathrm{UF} 1, \mathrm{Chroma}\right)$ is used to separate the 558- and 570-nm wavelengths. Another 25-m fiber (PM-S405-XP, Nufern) is used to delay the 570-nm pulse by 121 ns. A short-pass filter (SPF, 580SP, Omega) is placed at the output end of the $25-\mathrm{m}$ fiber to select the $570 \mathrm{~nm}$ wavelength.

The five wavelengths are combined using four beam combiners. First, a long-pass dichroic mirror $\left(\mathrm{DM}_{2}\right.$, T565lpxr-UF1, Chroma) is used to combine the 558- and 570-nm paths. Second, the $558 / 570-\mathrm{nm}$ beam is combined with the $620 / 640-\mathrm{nm}$ beam via a short-pass dichroic mirror $\left(\mathrm{DM}_{3}, \# 69-204\right.$, Edmund Optics). Third, a long-pass dichroic mirror ( $\mathrm{DM}_{4}, \mathrm{~T} 550 \mathrm{lpxr}$, Chroma) combines the 545-nm path and the 558/570/620/640-nm path. Last, the four SRS wavelengths are combined with the direct 532-nm path via a 10/90 BS and are coupled into the OR-PAM probe via a 2-m single-mode fiber (P1-460B-FC-2, Thorlabs Inc.).

The coupling efficiencies for all single-mode fibers are above $50 \%$. Because the 558-nm beam has high-order modes, when coupling it to the 2-m single-mode fiber, the coupling efficiency is $~ 30 \%$. In the 532-, 545-, 558-, and 570-nm light paths, the pulse energies on the sample surface are above $90 \mathrm{~nJ}$. The pulse energy for the 620/640-nm wavelength can reach $300 \mathrm{~nJ}$ on the sample surface. Detailed pulse energies and time delays for all wavelengths are summarized in Table 1. The shortest wavelength switching time is $121 \mathrm{~ns}$, which corresponds to $\sim 200-\mu \mathrm{m}$ imaging depth. This is acceptable for superficial imaging. For future deeper in vivo application, we may consider separating mixed signals from different wavelengths. For example, a deconvolution method has been developed to separate two overlapping signals generated from ultrafast dual-wavelength excitation. $^{47}$

In the OR-PAM probe, the laser beam from the 2-m fiber is focused by a pair of achromatic doublets (AC064-013-A, Thorlabs Inc.). The focused optical beam is reflected on an optical/acoustic beam combiner, is transmitted through a planoconcave lens (45-697, Edmund Optics), then illuminates the sample. Induced ultrasonic waves are collimated by the planoconcave lens, transmitted through the optical/acoustic beam combiner, and detected by a $50-\mathrm{MHz}$ broadband piezoelectric transducer (V214-BC-RM, Olympus). To optimize the detection sensitivity, the focused optical beam is coaxially and confocally aligned with the focused ultrasonic detection beam. The detailed information about the OR-PAM probe can be referred to in previous publications. ${ }^{46,48-50}$

\subsection{Laser Stability and Specifications}

Fiber coupling and SRS efficiency may be affected by airflow and temperature changes, causing random errors and long-term drift in the pulse energy. To improve the pulse-energy stability, we isolate the airflow using a cover and maintain the temperature fluctuation within $\pm 0.1^{\circ} \mathrm{C}$.

As shown in Table 1, we measured the pulse energy fluctuation and drift at each wavelength for one hour. The fluctuation is calculated from the standard deviation of the pulse energy. The drift is determined from the averaged pulse energy difference between the first and the last minute in the hour. With airflow isolation and temperature control, the pulse energy fluctuations at all wavelengths are reduced by $4 \%$ to $20 \%$, and the drifts for all wavelengths are reduced by $11 \%$ to $23 \%$. Because the pulse energy ratios among 532, 545, 558, and $570 \mathrm{~nm}$ are used in the calculation of blood flow and $\mathrm{sO}_{2}$, we list the relative pulse-energy fluctuations, i.e., the standard deviations of the pulse energy ratios of 545/532, 558/532, and 570/532, in Table 1 . The relative pulse energy fluctuations of 545, 558, and $570 \mathrm{~nm}$ are consistently smaller than their absolute pulse fluctuations. Because the MMF has a large mode area and is more sensitive to perturbation, the pulse energy fluctuations and drifts are larger in the multimode fiber $(558$ and $570 \mathrm{~nm})$ than in single-mode fibers (545 and 620/640 nm). With the airflow cover and temperature control, the pulse energy fluctuations of the SRS wavelengths (relative for 545, 558, and $570 \mathrm{~nm}$ and absolute for $620 / 640 \mathrm{~nm}$ ) are $26 \%$ to $41 \%$ higher than the pump wavelength, and the drifts are $20 \%$ to $75 \%$ higher than the pump one.

We measured the imaging depths for each wavelength by obliquely inserting a black human hair into fresh chicken breast tissue. The imaging depths are $0.75,0.76,0.77,0.78$, and $0.81 \mathrm{~mm}$ for $532,545,558,570$, and $620 / 640 \mathrm{~nm}$, respectively, as shown in the Figs. 2(a)-2(e). The lateral resolutions for the five wavelengths are measured by scanning the stainless-steel blade and measuring the amplitude profiles across the sharp edges. By fitting the PA signals to an edge spread function (ESF), we can obtain the line spread functions (LSFs) and the full widths at half maximum (FWHMs) as the lateral resolutions, as shown in Figs. 2(f)-2(j). The lateral resolutions are $3.12,3.25,3.37,3.51$, and $3.94 \mu \mathrm{m}$ for $532,545,558,570$, and $620 / 640 \mathrm{~nm}$, which agree with the simulation results shown as

Table 1 Pulse energy, delay time, pulse energy fluctuation, and drift of the five wavelengths. C\&T represents airflow isolation and temperature control.

\begin{tabular}{|c|c|c|c|c|c|c|c|c|}
\hline \multirow{2}{*}{$\begin{array}{l}\text { Wavelength } \\
(\mathrm{nm})\end{array}$} & \multirow{2}{*}{$\begin{array}{c}\text { Pulse } \\
\text { energy (nJ) }\end{array}$} & \multirow{2}{*}{$\begin{array}{l}\text { Delay } \\
\text { time (ns) }\end{array}$} & \multicolumn{2}{|c|}{$\begin{array}{c}\text { Pulse energy fluctuation } \\
\text { (SD) (\%) }\end{array}$} & \multicolumn{2}{|c|}{$\begin{array}{l}\text { Relative pulse energy } \\
\text { fluctuation (SD) (\%) }\end{array}$} & \multicolumn{2}{|c|}{$\begin{array}{l}\text { Pulse energy drift in } \\
\text { an hour (\%) }\end{array}$} \\
\hline & & & w/o C\&T & w C\&T & w/o C\&T & w C\&T & w/o C\&T & w C\&T \\
\hline 532 & 100 & 0 & 5.71 & 5.45 & - & - & 5.12 & 4.55 \\
\hline 545 & 100 & 139 & 8.22 & 7.15 & 7.78 & 7.01 & 7.63 & 6.04 \\
\hline 558 & 100 & 510 & 10.35 & 8.49 & 8.29 & 7.23 & 9.58 & 7.49 \\
\hline 570 & 90 & 631 & 10.98 & 8.78 & 8.95 & 7.68 & 10.42 & 7.96 \\
\hline $620 / 640$ & 300 & 260 & 7.54 & 6.85 & - & - & 6.70 & 5.45 \\
\hline
\end{tabular}



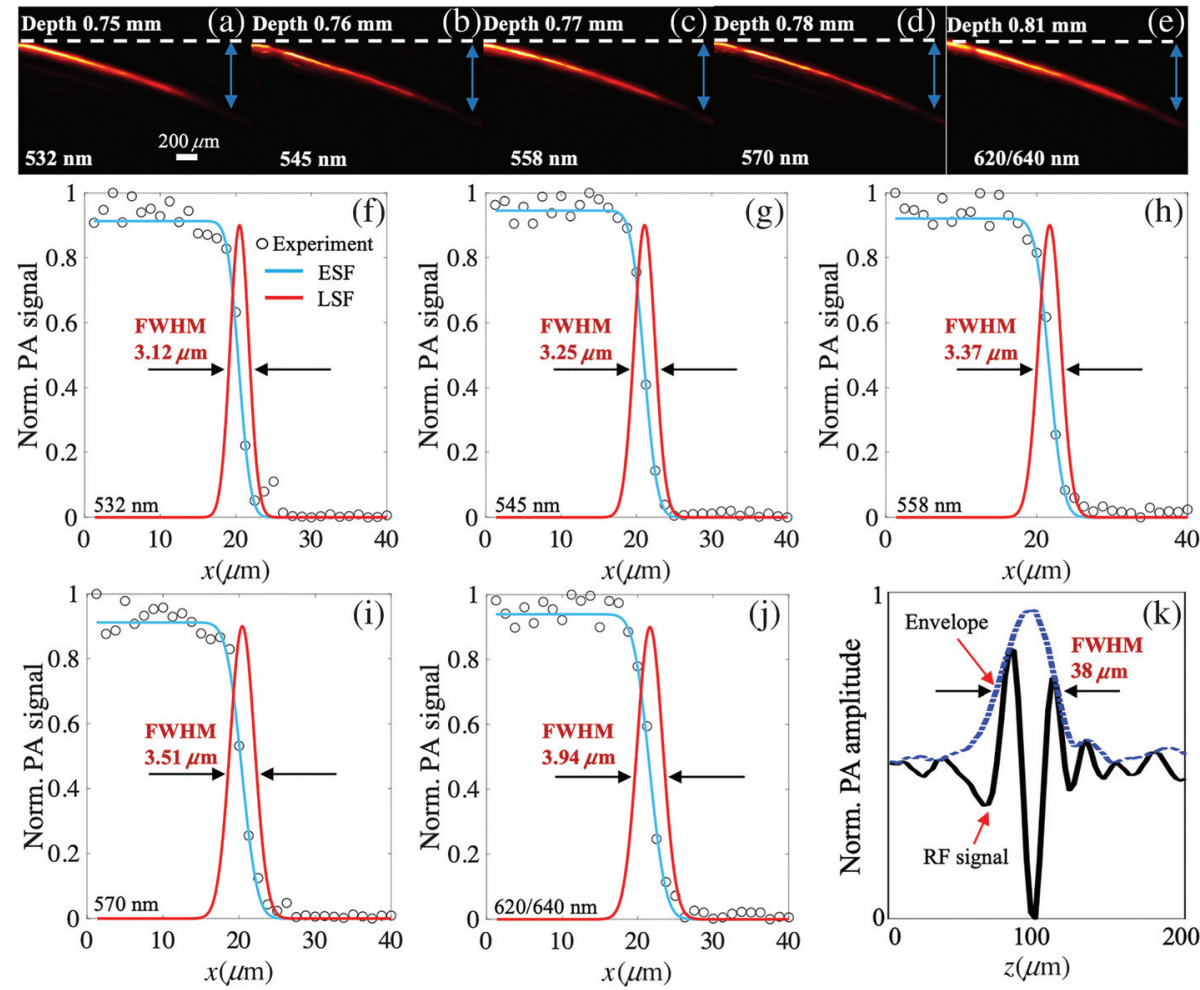

Fig. 2 (a)-(e) Measured penetration depths with 6-dB SNR for 532-, 545-, 558-, 570-, and $620 / 640-n m$ wavelengths. (f)-(j) Measured lateral resolutions of the five wavelengths. (k) Axial resolution measured via imaging a $10-\mu \mathrm{m}$-diameter tungsten filament.

Fig. S1 in the Supplementary Material. We also measured the axial resolutions by scanning a $10-\mu$ m-diameter tungsten filament. The FWHMs of a Hilbert-transformed A-line are shown in Fig. 2(k). The axial resolutions for all wavelengths are $38 \mu \mathrm{m}$.

\subsection{Multi-Contrast Imaging}

Figure 3(a) shows the laser spectrum measured with an optical spectrometer (USB 2000+, Ocean Optics). The linewidths of the SRS wavelengths are within $\pm 1.5 \mathrm{~nm}^{46,48}$ We use the molar extinction coefficients at the peak wavelengths for $\mathrm{sO}_{2}$ calculation. Figure 3(b) shows the absorption spectra of oxyhemoglobin, deoxyhemoglobin, and Evans blue (EB). ${ }^{51}$ The hemoglobin molecules and the EB dye have distinct absorption coefficients at 620 to $640 \mathrm{~nm}$. With EB dye uptake, the lymphatic vessels can be imaged with the $620 / 640-\mathrm{nm}$ wavelength. The other wavelengths are used to image the $C_{\mathrm{Hb}}, \mathrm{sO}_{2}$, and flow speed in the blood vessels.

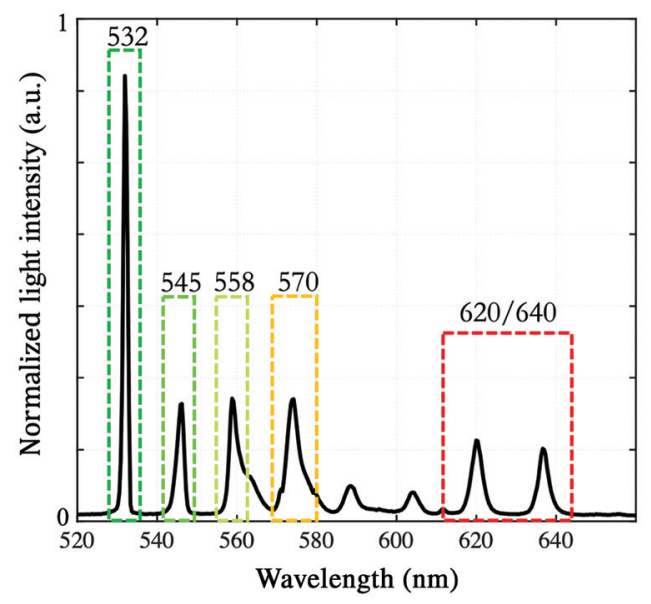

(a)

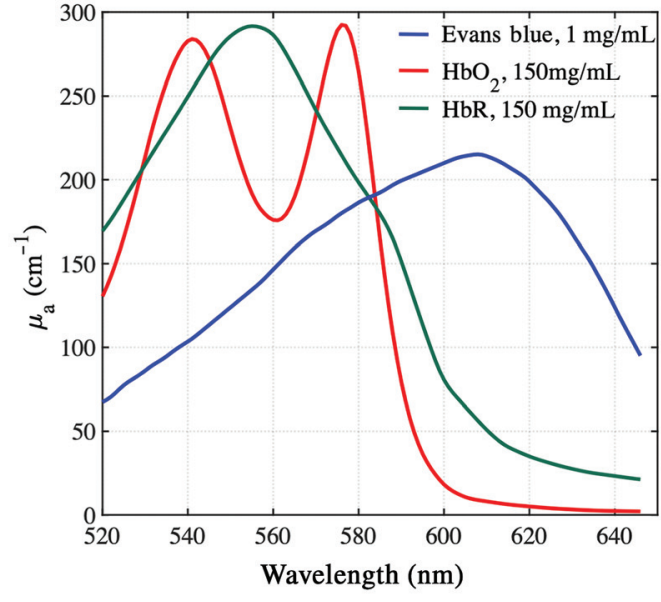

(b)

Fig. 3 (a) Spectrum of the five-wavelength stimulated-Raman-scattering laser. (b) Absorption spectra of $\mathrm{HbO}_{2}, \mathrm{HbR}$, and $\mathrm{EB}$. 
Under thermal and stress confinements, the PA amplitude in the blood vessel can be approximated as

$P=k \Gamma \eta F \mu_{a}, \mu_{a}=r C_{\mathrm{Hb}}\left[\mathrm{sO}_{2} \varepsilon^{\mathrm{oxy}}+\left(1-\mathrm{sO}_{2}\right) \varepsilon^{\mathrm{de}}\right]$,

where $k$ is a constant related to the PA detection sensitivity, $\Gamma$ is the local Grüneisen coefficient, $\eta$ is the efficiency of the absorbed optical energy that converts into heat, $F$ is the local optical fluence, $\mu_{a}$ is the absorption coefficient, $r$ is a constant, ${ }^{1}$ $C_{\mathrm{Hb}}$ is the total hemoglobin concentration, and $\varepsilon^{\text {oxy }}$ and $\varepsilon^{\mathrm{de}}$ are the molar extinction coefficients of $\mathrm{HbO}_{2}$ and $\mathrm{HbR}$ at the excitation wavelength, respectively.

At the 532-nm wavelength, $\varepsilon^{\text {oxy }}$ and $\varepsilon^{\text {de }}$ are almost the same, thus $\mu_{a}$ is not sensitive to the $\mathrm{sO}_{2}$ change..$^{52}$ We can determine the relative change of $C_{\mathrm{Hb}}$ from the PA amplitude at $532 \mathrm{~nm}$ or the absolute $C_{\mathrm{Hb}}$ after calibration.

The 545- and 532-nm wavelengths are used to measure the blood flow speed using a dual-pulse photoacoustic flowmetric method that is published in Ref. 46. When we excite two PA signals with a sub-microseconds delay, the residual heat from the first pulsed excitation elevates the second PA amplitude via the Grüneisen relaxation effect. ${ }^{53}$ Blood flow may accelerate the heat dissipation and thus alter the second PA amplitude as follows:

$P_{2}=r_{1} \frac{F_{2}}{F_{1}} P_{1}+r_{1} \frac{F_{2}}{F_{1}} P_{1}^{2} A e^{-\left(\tau_{\alpha}+b v\right) \delta t}$,

where $r_{1}=\frac{\mu_{a 2}}{\mu_{a 1}}, P_{1,2}$ and $F_{1,2}$ are the PA amplitudes and the optical fluences at $\lambda_{1}=532 \mathrm{~nm}$ and $\lambda_{2}=545 \mathrm{~nm}, \delta t$ is the time delay between the 532- and 545-nm laser pulses, and $A, \tau_{\alpha}$, and $b$ can be determined via calibration. ${ }^{46}$ The detailed calibration method is shown as part 2 in the Supplementary Material. Here, $A, \tau_{\alpha}$, and $b$ were calibrated as $0.09 \pm 0.04$ (SD), $9.68 \pm 0.85$ (SD), and $0.25 \pm 0.06$ (SD). Via measuring the two PA signals at the 532- and 545-nm wavelengths (two isosbestic points), we can determine the blood flow speed using only two A-lines. After measuring the vessel diameter and the average flow speed, we can further determine the blood flow rate. ${ }^{54-56}$ The detailed calculation method is shown as Eq. S(1) in the Supplementary Material.

Using 532-, 545-, 558-, and 570-nm wavelengths, we can measure oxygen saturation. Considering the Grüneisen relaxation effect among successive pulse excitations, a PA amplitude is affected by previous excitations. Because the 620/640-nm PA signal (the third pulse) from the blood vessel is only $\sim 2.5 \%$ of those by other pulses, we neglect the heating by the third pulse. The PA amplitudes for the first, second, fourth, and fifth pulses can be written as

$$
\begin{aligned}
P_{1}= & k \Gamma_{0} \eta F_{1} \mu_{a_{1}}, \\
P_{2}= & k \Gamma_{0} \eta F_{2} \mu_{a_{2}}\left[1+A P_{1} e^{-\left(\tau_{\alpha}+b v\right) \delta t_{12}}\right] \\
P_{4}= & k \Gamma_{0} \eta F_{4} \mu_{a_{4}}\left[1+A P_{1} e^{-\left(\tau_{\alpha}+b v\right) \delta t_{14}}+A P_{1} \frac{F_{2} \mu_{a 2}}{F_{1} \mu_{a 1}} e^{-\left(\tau_{\alpha}+b v\right) \delta t_{24}}\right] \\
P_{5}= & k \Gamma_{0} \eta F_{5} \mu_{a_{5}}\left[1+A P_{1} e^{-\left(\tau_{\alpha}+b v\right) \delta t_{15}}+A P_{1} \frac{F_{2} \mu_{a 2}}{F_{1} \mu_{a 1}} e^{-\left(\tau_{\alpha}+b v\right) \delta t_{25}}\right. \\
& \left.+A P_{1} \frac{F_{4} \mu_{a 4}}{F_{1} \mu_{a 1}} e^{-\left(\tau_{\alpha}+b v\right) \delta t_{45}}\right]
\end{aligned}
$$

where $\delta t_{i j}$ is the time interval between the $i$ 'th and $j$ 'th pulses. With the calibrated $A, \tau_{\alpha}$, and $b$, and determined flow speed $v$, we can solve the above equations and determine the $\mathrm{sO}_{2}$ value from the four measurements.

\section{Results}

\subsection{In Vivo Imaging of Blood and Lymphatic Vessels}

Four-week-old female ICR mice were used in experiments. All procedures involving animals were approved by the animal ethical committee of the City University of Hong Kong. The mice were anesthetized by inhaling isoflurane gas. EB dye solution $(0.5 \%$, mass fraction, $20 \mu L)$ was injected into the ear tip to label the lymphatic vessels. The mouse ear was placed on a flat holder and was imaged in 5 min after EB injection. The laser repetition rate is $4 \mathrm{kHz}$ for each wavelength. The pulse energy is $160 \mathrm{~nJ}$ for the $620 / 640-\mathrm{nm}$ wavelength and $90 \mathrm{~nJ}$ for the other four wavelengths. The scanning step size is $2.5 \mu \mathrm{m}$. The imaging area is $2.5 \mathrm{~mm} \times 2.5 \mathrm{~mm}$. It takes $250 \mathrm{~s}$ to acquire a volumetric image. Maximum-amplitude-projected images are shown in Fig. 4. Figure 4(a) is the $\mathrm{sO}_{2}$ image. The $\mathrm{sO}_{2}$ images without and with compensation for the Grüneisen relaxation effect are shown as Fig. S2 in the Supplementary Material. The average $\mathrm{sO}_{2}$ values calculated with and without compensation for the Grüneisen relaxation effect are significantly different $(P<0.001)$. With compensation, the $\mathrm{sO}_{2}$ values in the arteries and veins are closer to their normal physiological values. ${ }^{19,20,23,46}$ The compensation for the Grüneisen relaxation improves the averaged $\mathrm{sO}_{2}$ values by $4 \%$ to $17 \%$ in the arteries and by $21 \%$ to $24 \%$ in the veins. The $\mathrm{sO}_{2}$ in the veins is corrected more than that in the arteries. The possible reason is that the vein has slower flow speed and thus has a more severe Grüneisen relaxation effect than the arteries. Due to the same reason, the $\mathrm{sO}_{2}$ is corrected more in the small arteries than in the big ones. Because the flow speed and the Grüneisen relaxation effect in the veins are relatively uniform, the $\mathrm{sO}_{2}$ correction shows no obvious difference in the big and small venous vessels. Figure 4(b) shows the blood flow speed image. Figure 4(d) depicts the average $\mathrm{sO}_{2}$ and flow speeds in different vessel segments. From the root to the tip of the mouse ear, the averaged blood flow speed in the trunk arteries decreases from $5.8 \pm 0.24$ (SD) to $5.0 \pm 0.21$ (SD) mm/s. In the small arterial branches, the averaged blood flow speed decreases to $3.4 \pm 0.36(\mathrm{SD}) \mathrm{mm} / \mathrm{s}$. The averaged blood flow speed in the veins maintains at $\sim 1.9 \pm 0.18 \mathrm{~mm} / \mathrm{s}$. After measuring the blood vessel diameter, we can further compute the flow rate. In Fig. 4(b), the profiles across five arteries and six veins are labeled with white dashed lines as $\mathrm{A}_{1-5}$, and $\mathrm{V}_{1-6}$, respectively. As shown in Fig. 4(e), the vessel diameters are 86, 74, 58, 47, and $43 \mu \mathrm{m}$ for $\mathrm{A}_{1-5}$ and $189,112,97,84,48$, and $123 \mu \mathrm{m}$ for $\mathrm{V}_{1-6}$. The corresponding flow rates are $1.03,0.65,0.41,0.25$, and $0.15 \mu \mathrm{L} / \mathrm{min}$ for $\mathrm{A}_{1-5}$ and $1.68,0.63,0.42,0.28,0.14$, and $0.68 \mu \mathrm{L} / \mathrm{min}$ for $\mathrm{V}_{1-6}$. The veins $\mathrm{V}_{2}, \mathrm{~V}_{3}$, and $\mathrm{V}_{6}$ are converged to $\mathrm{V}_{1}$, and the total flow rate in $\mathrm{V}_{2}, \mathrm{~V}_{3}$, and $\mathrm{V}_{6}$ matches the flow rate in $V_{1}$. The total flow rate in $V_{4}$ and $V_{5}$ is approximately equal to the one in $V_{3}$. Similarly, the arteries $A_{2}$ and $A_{3}$ are the branches of $A_{1}$, and thus the total flow rate in $A_{2}$ and $A_{3}$ matches the one in $A_{1}$. The total flow rate in $A_{4}$ and $A_{5}$ matches the flow rate in $A_{3}$. Four pairs of arteries and veins $\left(A_{2-5}\right.$ and $\mathrm{V}_{2-5}$ ) are compared. The flow rates in the arteries match the ones in the veins. The flow rates in $A_{1}$ and $V_{1}$ are not very close. We guess the possible reason might be that the two vessels are different in shape, and the measured diameter cannot be used to accurately estimate the cross-sectional area. Figures 4(c) and 4(d) show the blood and lymphatic vessels and the relative 

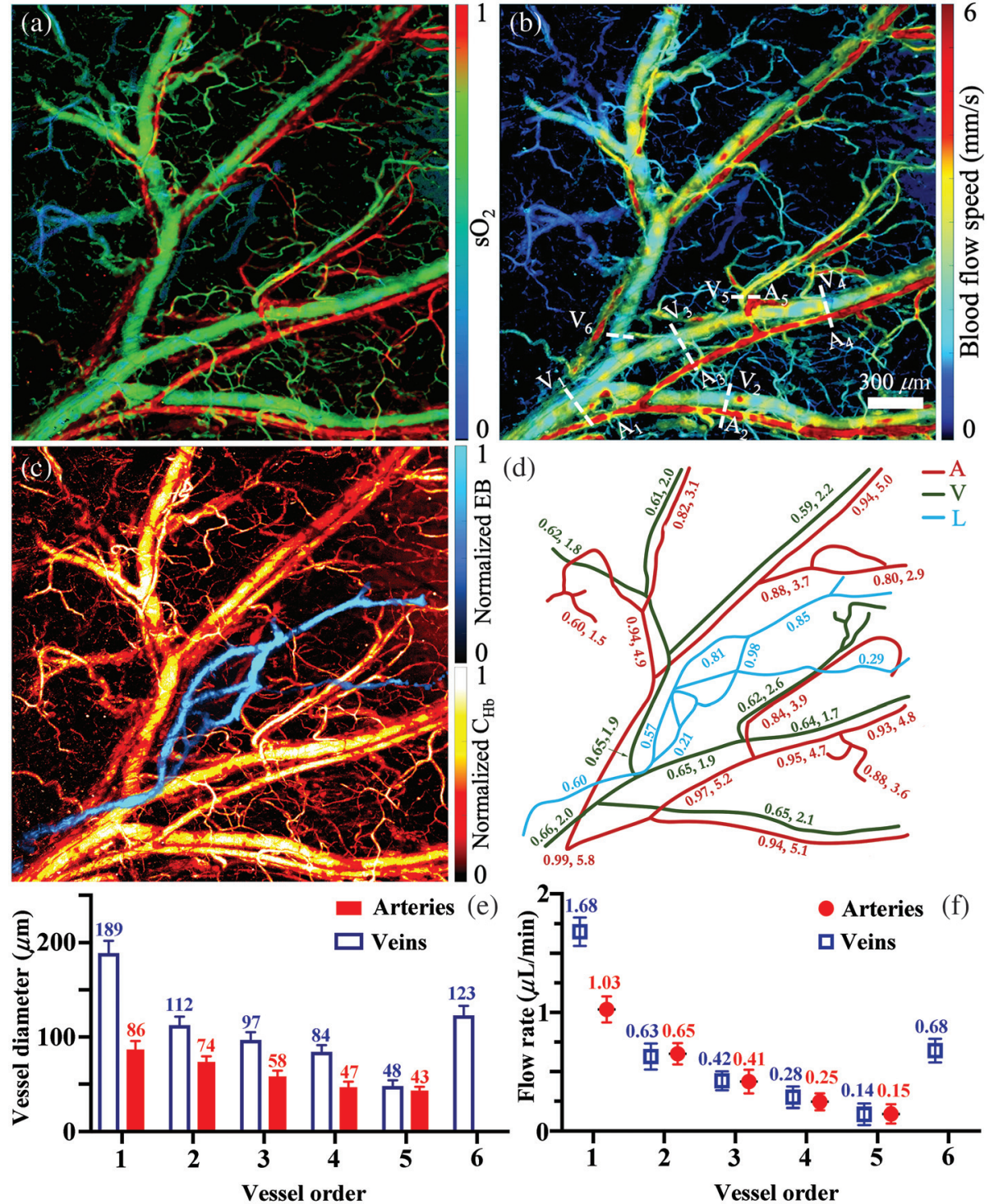

Fig. 4 Five-wavelength OR-PAM of the blood and lymphatic vessels in the mouse ear. The imaging area is $2.5 \mathrm{~mm} \times 2.5 \mathrm{~mm}$. (a) Compensated oxygen saturation. (b) Blood flow speed determined with the dual-pulse method. The profiles of five arteries and six veins are labeled with white dashed lines. (c) PAM imaging of the blood and lymphatic vessels. (d) Variation of oxygen saturation, blood flow speed, and relative lymphatic concentration from the root to the tip of the mouse ear. A, arteries; $V$, veins; $L$, lymphatic vessels. Along the arteries and veins, the first value is compensated oxygen saturation, and the second value is blood flow speed $(\mathrm{mm} / \mathrm{s})$. (e) The averaged diameter $(\mu \mathrm{m})$ of arteries and veins profiles labeled in (b). (f) The averaged flow rate $(\mu L / \mathrm{min})$ of arteries and veins profiles labeled in (b). Data are presented as mean $\pm S D$, and the mean values are labeled in the figure.

lymphatic concentration in vessel segments. With distinct absorption spectra, the blood and the dye-labeled lymphatic vessels can be identified with high contrast. The highest SNRs (signal amplitude over noise standard deviation) of the blood and lymphatic vessels are 20.1 and $22.3 \mathrm{~dB}$.

\subsection{In Vivo Imaging of Tumor, Lymphatic Clearance, and Brain Function}

To demonstrate the potential applications, we first conducted in vivo early cancer detection experiments using five-wavelength
OR-PAM. Before tumor implantation, the multifunctional vascular images of hemoglobin concentration, compensated oxygen saturation, and blood flow speed were acquired in the mouse ear and are shown as Fig. S3 in the Supplementary Material. Then, 4T1 breast cancer cells were injected to the mouse ear. After the tumor developed for 5 days, the same region was imaged to demonstrate the changes of the aforementioned multi-functional parameters. As a hallmark of cancer, ${ }^{57,58}$ obvious angiogenesis is observed in Fig. 5(a). Figures 5(b) and 5(c) show the $\mathrm{sO}_{2}$ and blood flow speed in the tumor region. Compared with the baseline, the averaged $\mathrm{sO}_{2}$ in the tumor 

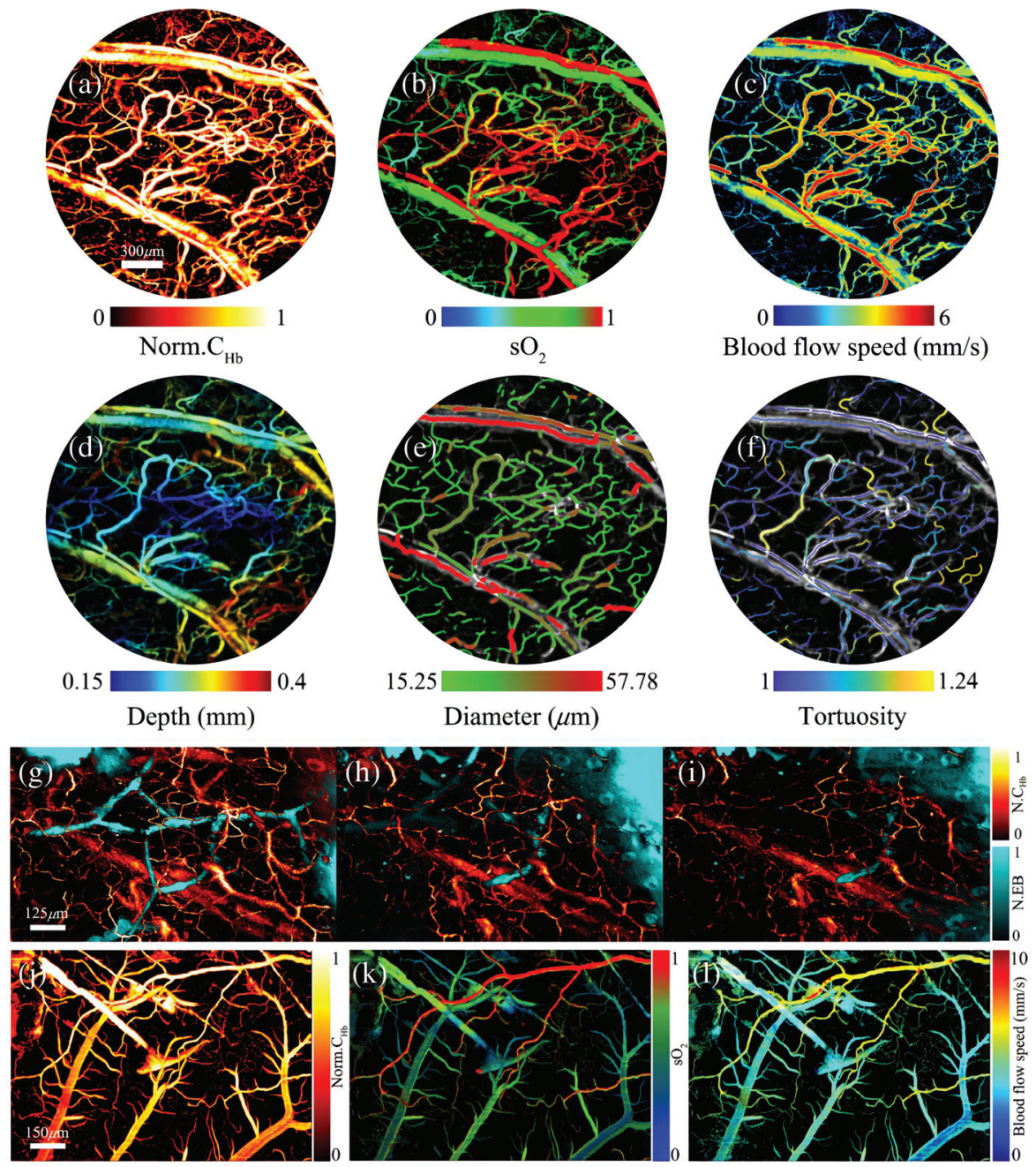

Fig. 5 (a)-(f) OR-PAM of hemoglobin concentration, oxygen saturation, blood flow speed, depth, diameter, and tortuosity in the tumor region. (g)-(i) Simultaneous imaging of hemoglobin and dye concentrations at 0,10 , and 20 min after EB dye injection. (j)-(I) In vivo brain imaging of hemoglobin concentration, oxygen saturation, and blood flow speed.

vessels increases by $45 \%$ to $51 \%$, and the blood flow speed increases by $32 \%$ to $37 \%$, indicating hyperoxia and elevated blood perfusion in the early-stage tumor due to fast growing. ${ }^{55,57,58}$ Figure 5(d) shows pseudo-color-encoded vascular depth, manifesting height changes in the tumor region. Figures 5(e) and 5(f) show the vessel dilation and vessel tortuosity in the tumor region. Compared with the non-tumor region, the average diameter and tortuosity of the venules are increased by $48 \%$ to $52 \%$ and $10 \%$ to $12 \%$ in the tumor region, which has been validated in other reports. ${ }^{57,58}$ Detailed quantification methods and comparisons are shown as Fig. S4 in the Supplementary Material.

Enabled by the new wavelength, five-wavelength OR-PAM can image the dye-removal processes in lymphatic vessels. Figures 5(g)-5(i) show the simultaneous imaging of the blood and the dye-labeled lymphatic vessels in the mouse ear at 0,10 , and 20 min after injecting EB dye solution $(0.5 \%$, mass fraction, $20 \mu L)$. The normalized concentrations of hemoglobin and $\mathrm{EB}$ are encoded with red and blue colors, respectively. After $20 \mathrm{~min}$, the average EB concentration decreases by $87 \%$ in the lymphatic vessels, indicating the waste clearance ability of the local lymphatic vessels. Taking advantage of simultaneous multi-contrast imaging, five-wavelength OR-PAM can realize the simultaneous in vivo dye-labeled lymphatic and multi-functional vascular imaging, which is of great importance to imaging the dynamic blood perfusion and lymphatic circulation.

We also demonstrated five-wavelength OR-PAM in multicontrast brain imaging. A $2 \mathrm{~mm} \times 2 \mathrm{~mm}$ window was opened in the mouse skull after the mouse was anesthetized. Using 60-nJ pulse energy, we acquired functional brain images and quantified normalized hemoglobin concentration, $\mathrm{sO}_{2}$, and blood flow speed, as shown in Figs. 5(j)-5(1). In the arteries and veins, the average $\mathrm{sO}_{2}$ values are $0.95 \pm 0.06(\mathrm{SD})$ and $0.68 \pm 0.08$ (SD); the average blood flow speeds are $7.2 \pm 0.62(\mathrm{SD}) \mathrm{mm} / \mathrm{s}$ and $3.9 \pm 0.45(\mathrm{SD}) \mathrm{mm} / \mathrm{s}$, respectively. Because the new fivewavelength system has red light, other exogenous photoacoustic contrast agents can be used in the future to label neurons or tumors in the brain.

\section{Conclusion}

We developed five-wavelength OR-PAM that can simultaneously image the EB-labeled lymphatic vessel, as well as 
hemodynamic and functional information in the blood vessel in a single scanning. A five-wavelength pulsed laser was developed based on the SRS effect. Fiber-based optical delay switches the wavelengths within sub-microseconds, which reduces the misalignment in multi-wavelength imaging. We isolate airflow and control the temperature of the SRS laser to further stabilize the pulse energy. The 532-, 545-, 558-, and $570-\mathrm{nm}$ wavelengths are suitable for blood vessel imaging, and the 620/640-nm wavelength offers high contrast for lymphatic vessel imaging. With the blood flow speed measured by the dual-pulse flowmetric method and corresponding vessel diameter, the blood flow rates in several arteries and veins were estimated, and the results manifest the flow conservation. In vivo experiments demonstrated the simultaneous multicontrast imaging of blood and lymphatic vessels. We showed the potential applications of this technique in early cancer detection, monitoring lymphatic circulation, and imaging the brain. Other molecular or nano-probes that have strong absorption at $620 / 640 \mathrm{~nm}$ can also be used as the contrast agent in the future. Co-registered hemodynamic, functional, and molecular information can be acquired in a single scanning. This technical advance enables simultaneous multi-contrast imaging in the living body, offering a new biomedical imaging tool.

\section{Acknowledgments}

This work was partially supported by the National Natural Science Foundation of China (NSFC) (Nos. 81627805 and 61805102); Research Grants Council of the Hong Kong Special Administrative Region (Nos. 21205016, 11215817, and 11101618); Shenzhen Basic Research Project (No. JCYJ20170413140519030). We thank Dr. Xiangwei Lin for helping inject the dye. The authors declare that there are no conflicts of interest related to this article.

\section{References}

1. R. A. Hyde et al., "Vasculature and lymphatic system imaging and ablation associated with a reservoir," US Patent US20090093728A1 (9 October 2012).

2. L. A. Fordham, C. J. Chung, and L. F. Donnelly, "Imaging of congenital vascular and lymphatic anomalies of the head and neck," Neuroimaging Clin. N. Am. 10(1), 117-136 (2000).

3. T. Hoshida et al., "Imaging steps of lymphatic metastasis reveals that vascular endothelial growth factor-C increases metastasis by increasing delivery of cancer cells to lymph nodes: therapeutic implications," Cancer Res. 66(16), 8065-8075 (2006).

4. C. Martel et al., "Photoacoustic lymphatic imaging with high spatial-temporal resolution," J. Biomed. Opt. 19(11), 116009 (2014).

5. G. Follain et al., "Hemodynamic forces tune the arrest, adhesion, and extravasation of circulating tumor cells," Dev. Cell 45(1), 33-52.e12 (2018).

6. K. Yttersian Sletta et al., "Oxygen-dependent regulation of tumor growth and metastasis in human breast cancer xenografts," PLoS ONE 12(8), e0183254 (2017).

7. D. M. Lewis et al., "Intratumoral oxygen gradients mediate sarcoma cell invasion," Proc. Natl. Acad. Sci. U. S. A. 113(33), 9292-9297 (2016).

8. A. R. Nobre et al., "The different routes to metastasis via hypoxiaregulated programs," Trends Cell Biol. 28(11), 941-956 (2018).

9. H. Kobayashi et al., "Simultaneous multicolor imaging of five different lymphatic basins using quantum dots," Nano Lett. 7(6), 1711-1716 (2007).
10. T. Barrett, P. L. Choyke, and H. Kobayashi, "Imaging of the lymphatic system: new horizons," Contrast Media Mol. Imaging 1(6), 230-245 (2006).

11. F. Ogata et al., "Novel lymphography using indocyanine green dye for near-infrared fluorescence labeling," Ann. Plast. Surg. 58(6), 652-655 (2007).

12. S. Kim et al., "Near-Infrared fluorescent type II quantum dots for sentinel lymph node mapping," Nat. Biotechnol. 22(1), 93-97 (2004).

13. R. Guimaraes et al., "MR lymphography with superparamagnetic iron nanoparticles in rats: pathologic basis for contrast enhancement," Am. J. Roentgenol. 162(1), 201-207 (1994).

14. H. Kobayashi et al., "Multicolor imaging of lymphatic function with two nanomaterials: quantum dot-labeled cancer cells and dendrimer-based optical agents," Nanomedicine 4(4), 411-419 (2009).

15. C. Kim et al., "Sentinel lymph nodes and lymphatic vessels: noninvasive dual-modality in vivo mapping by using indocyanine green in rats-volumetric spectroscopic photoacoustic imaging and planar fluorescence imaging," Radiology 255(2), 442-450 (2010).

16. K. H. Song et al., "Noninvasive photoacoustic identification of sentinel lymph nodes containing methylene blue in vivo in a rat model," J. Biomed. Opt. 13(5), 054033 (2008).

17. K. H. Song et al., "Near-Infrared gold nanocages as a new class of tracers for photoacoustic sentinel lymph node mapping on a rat model," Nano Lett. 9(1), 183-188 (2009).

18. K. H. Song et al., "Noninvasive in vivo spectroscopic nanorodcontrast photoacoustic mapping of sentinel lymph nodes," Eur. J. Radiol. 70(2), 227-231 (2009).

19. L. V. Wang and $\mathrm{H}$. Wu, Biomedical Optics: Principles and Imaging, John Wiley \& Sons (2012).

20. S. Hu, "Optical-resolution photoacoustic microscopy," $\mathrm{PhD}$ Thesis, Washington University (2010).

21. L. V. Wang and J. Yao, "A practical guide to photoacoustic tomography in the life sciences," Nat. Methods 13(8), 627-638 (2016).

22. J. Yao and L. V. Wang, "Photoacoustic microscopy," Laser Photonics Rev. 7(5), 758-778 (2013).

23. C. Kim, C. Favazza, and L. V. Wang, "In vivo photoacoustic tomography of chemicals: high-resolution functional and molecular optical imaging at new depths," Chem. Rev. 110(5), 2756-2782 (2010).

24. S. Jeon et al., "Real-time delay-multiply-and-sum beamforming with coherence factor for in vivo clinical photoacoustic imaging of humans," Photoacoustics 15, 100136 (2019).

25. H.-C. Zhou et al., "Optical-resolution photoacoustic microscopy for monitoring vascular normalization during anti-angiogenic therapy," Photoacoustics 15, 100143 (2019).

26. Y. Zhou et al., "Single-shot linear dichroism optical-resolution photoacoustic microscopy," Photoacoustics 16, 100148 (2019).

27. M. Li, Y. Tang, and J. Yao, "Photoacoustic tomography of blood oxygenation: a mini review," Photoacoustics 10, 65-73 (2018).

28. J. Chen et al., "Wide-field polygon-scanning photoacoustic microscopy of oxygen saturation at $1-\mathrm{MHz}$ A-line rate," Photoacoustics 20, 100195 (2020).

29. D. Li et al., "Micro-rocket robot with all-optic actuating and tracking in blood," Light Sci. Appl. 9(1), 84 (2020).

30. Y. Zhang and L. Wang, "Video-rate ring-array ultrasound and photoacoustic tomography," IEEE Trans. Med. Imaging 39(12), 4369-4375 (2020).

31. C. Yin et al., "Organic semiconducting polymer nanoparticles for photoacoustic labeling and tracking of stem cells in the second near-infrared window," ACS Nano 12(12), 12201-12211 (2018).

32. D. Wang et al., "Nonlinear photoacoustic imaging by in situ multiphoton upconversion and energy transfer," ACS Photonics 4(11), 2699-2705 (2017).

33. M. Pramanik et al., "In vivo carbon nanotube-enhanced noninvasive photoacoustic mapping of the sentinel lymph node," Phys. Med. Biol. 54(11), 3291-3301 (2009). 
34. Z. Chen et al., "Performance of optoacoustic and fluorescence imaging in detecting deep-seated fluorescent agents," Biomed. Opt. Express 9(5), 2229-2239 (2018).

35. R. J. Zemp et al., "Photoacoustic imaging of the microvasculature with a high-frequency ultrasound array transducer," J. Biomed. Opt. 12(1), 010501 (2007).

36. M. Erfanzadeh and Q. Zhu, "Photoacoustic imaging with low-cost sources; a review," Photoacoustics 14, 1-11 (2019).

37. D. Razansky et al., "Multispectral opto-acoustic tomography of deep-seated fluorescent proteins in vivo," Nat. Photonics 3(7), 412-417 (2009).

38. P. Beard, "Biomedical photoacoustic imaging," Interface Focus 1(4), 602-631 (2011).

39. D. Razansky, A. Buehler, and V. Ntziachristos, "Volumetric realtime multispectral optoacoustic tomography of biomarkers," Nat. Protoc. 6(8), 1121-1129 (2011).

40. A. Taruttis and V. Ntziachristos, "Advances in real-time multispectral optoacoustic imaging and its applications," Nat. Photonics 9(4), 219-227 (2015)

41. H. F. Zhang, K. Maslov, and L. V. Wang, "In vivo imaging of subcutaneous structures using functional photoacoustic microscopy," Nat. Protoc. 2(4), 797-804 (2007).

42. T. Jin et al., "Portable optical resolution photoacoustic microscopy (pORPAM) for human oral imaging," Opt. Lett. 42(21), 4434-4437 (2017).

43. Q. Chen et al., "Ultracompact high-resolution photoacoustic microscopy," Opt. Lett. 43(7), 1615-1618 (2018).

44. P. Hajireza, A. Forbrich, and R. J. Zemp, "Multifocus opticalresolution photoacoustic microscopy using stimulated Raman scattering and chromatic aberration," Opt. Lett. 38(15), 27112713 (2013).

45. S. H. Baek and W. B. Roh, "Single-mode Raman fiber laser based on a multimode fiber," Opt. Lett. 29(2), 153-155 (2004).

46. C. Liu, Y. Liang, and L. Wang, "Single-shot photoacoustic microscopy of hemoglobin concentration, oxygen saturation, and blood flow in sub-microseconds," Photoacoustics 17, 100156 (2020).

47. Y. Zhou et al., "Optical-resolution photoacoustic microscopy with ultrafast dual-wavelength excitation," J. Biophotonics 13(6), e201960229 (2020).

48. C. Liu, Y. Liang, and L. Wang, "Optical-resolution photoacoustic microscopy of oxygen saturation with nonlinear compensation," Biomed. Opt. Express 10(6), 3061-3069 (2019).

49. L. Wang et al., "Fast voice-coil scanning optical-resolution photoacoustic microscopy," Opt. Lett. 36(2), 139-141 (2011).

50. L. Wang, K. Maslov, and L. V. Wang, "Single-cell label-free photoacoustic flowoxigraphy in vivo," Proc. Natl. Acad. Sci. U. S. A. 110(15), 5759-5764 (2013).

51. M. Damodaran, A. Amelink, and J. F. De Boer, "Optimal wavelengths for subdiffuse scanning laser oximetry of the human retina," J. Biomed. Opt. 23(8), 086003 (2018).
52. R. J. Hill and W. Koningsberg, "The structure of human hemoglobin," J. Biol. Chem. 237(10), 3151-3156 (1962).

53. L. Wang, C. Zhang, and L. V. Wang, "Grüneisen relaxation photoacoustic microscopy," Phys. Rev. Lett. 113(17), 174301 (2014).

54. A. Y. Shih et al., "Active dilation of penetrating arterioles restores red blood cell flux to penumbral neocortex after focal stroke," J. Cereb. Blood Flow Metab. 29(4), 738-751 (2009).

55. J. Yao et al., "Label-free oxygen-metabolic photoacoustic microscopy in vivo," J. Biomed. Opt. 16(7), 076003 (2011).

56. R. Cao et al., "Functional and oxygen-metabolic photoacoustic microscopy of the awake mouse brain," NeuroImage 150, 77-87 (2017).

57. P. Vaupel, F. Kallinowski, and P. Okunieff, "Blood flow, oxygen and nutrient supply, and metabolic microenvironment of human tumors: a review," Cancer Res. 49(23), 6449-6465 (1989).

58. J. Chen et al., "Confocal visible/NIR photoacoustic microscopy of tumors with structural, functional, and nanoprobe contrasts," Photonics Res. 8(12), 1875-1880 (2020).

Chao Liu received his $\mathrm{PhD}$ in biomedical engineering at City University of Hong Kong. He received his MS degree in electronic engineering from Hong Kong University of Science and Technology and BS degree in automation from Xi'an Jiaotong University. His current research areas are biomedical photonics, multi-contrast OR-PAM, and PA-related applications.

Jiangbo Chen is a PhD student in the Department of Biomedical Engineering, City University of Hong Kong. He received his bachelor's degree from the Northeast Forestry University and his master's degree from Harbin Institute of Technology. His research focuses on biophotonics and biomedical imaging.

Yachao Zhang received his PhD from the Biomedical Engineering Department, City University of Hong Kong. He received his BS degree from Jilin University. His research focuses on photoacoustic computed tomography system development, photoacoustic image reconstruction and processing, and high intensity focused ultrasound therapy.

Jingyi Zhu received her MS degree in biomedical engineering at Zhejiang University and BS degree in electrical engineering from Zhengzhou University. Her current research areas are biophotonics, multi-scale PAM, and PA-related applications.

Lidai Wang is an associate professor in the Department of Biomedical Engineering, City University of Hong Kong. He received his BS and MS degrees from the Tsinghua University, Beijing and his PhD from the University of Toronto, Canada. His research focuses on biophotonics, biomedical imaging, wavefront engineering, instrumentation, and their biomedical applications. 